\title{
Standardizing the Unit of Measurements in LOINC-Coded Laboratory Tests Can Significantly Improve Semantic Interoperability
}

\author{
Abdul Mateen RAJPUT ${ }^{\mathrm{a}, 1}$, Sarah BALLOUT ${ }^{\mathrm{b}}$ and Cora DRENKHAHN ${ }^{\mathrm{c}, \mathrm{d}}$

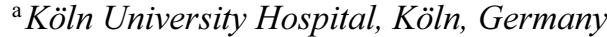 \\ ${ }^{\mathrm{b}}$ Peter L. Reichertz Institute for Medical Informatics of TU Braunschweig and \\ Hannover Medical School, Hannover, Germany \\ ' IT Center for Clinical Research (ITCR-L), University of Lübeck, Germany \\ dinstitute of Medical Informatics (IMI), University of Lübeck, Germany
}

Keywords. Semantic Interoperability, LOINC, UCUM

\section{Introduction}

Within healthcare environments, diagnostic and clinical data comes from many different systems, which frequently leads to an inconsistent presentation of important information. Controlled standard terminologies such as Logical Observation Identifiers Names and Codes (LOINC), allow some of the data inconsistency problems to be resolved. In the German Medical Informatics Initiative (MI-I), LOINC is used for sharing laboratory data across different departments and different university hospitals [1], [2]. Therefore, a list of 300 frequently used LOINC codes was composed, enabling a common basis for the mapping of site-specific terms and measurements to LOINC at all participating sites.

Despite being a standardized coding system LOINC has been shown to leave some ambiguity in the tests coded with it, particularly by defining a "kind of quantity/property" instead of the unit of measurement itself [3], as shown in Table 1. In this short paper inter-mapping variability arising from the ambiguous property definition is investigated.

Table 1. Two different LOINC Terms for similar measurements, differing only in their specified property.

\begin{tabular}{rcc}
\hline LOINC Code & \multicolumn{1}{c}{ LOINC Term } & Example Unit \\
\hline $2345-7$ & Glucose [Mass/volume] in Serum or Plasma & $\mathrm{mg} / \mathrm{dL}$ \\
$14749-6$ & Glucose [Moles/volume] in Serum or Plasma & $\mathrm{mmol} / \mathrm{L}$ \\
\hline
\end{tabular}

\section{Methods}

In the HiGHmed consortium participating university hospitals are required to map their local laboratory terms to LOINC based on the agreed TOP300 list. The mapping was

${ }^{1}$ Corresponding Author, Abdul-Mateen Rajput, Universitätsklinikum Köln, Kerpener Str. 62, 50937 Köln, Germany; E-mail: Abdul.mateen@uni-koeln.de. 
done by domain experts, either using RELMA [4] or the LOINC web interface. Afterwards, site-specific mapping tables, with different locally used naming conventions, were joined by using Inner Join so only matching rows were included in further work.

The unique identifiers were LOINC codes, so we could identify different test names and additional information including the unit of measurement that were associated with the same concepts. Entries were evaluated for discrepancies which were further analyzed.

\section{Results}

Conflicts in the resulting table were found both for the name and also for the unit of measurements assigned to the same code. For 118 out of 186 entries the same unit was defined at both sites, whereas 67 disparities could be divided into two categories:

1) Different laboratories used two slightly different versions of the same unit of measurements. Table 2 shows "sec" and "sek" being used for time points.

2) The unit is reported in different granularities e.g. gram per liter versus milligram per milliliter. Examples can be found in rows two and three of Table 2.

Table 2. Examples of disparate site-specific annotations mapped to the same LOINC

\begin{tabular}{rclrr}
\hline LOINC Code & Name (Site 1) & \multicolumn{1}{c}{ Name (Site 2) } & Unit (Site 1) & Unit (Site 2) \\
\hline $3243-3$ & Thrombinzeit & Thrombinzeit (CP) & $\mathrm{sek}$ & $\mathrm{sec}$ \\
$3013-0$ & Thyreoglob., hTG & Thyreoglobulin (S) & $\mu \mathrm{g} / \mathrm{l}$ & $\mathrm{ng} / \mathrm{ml}$ \\
$19113-0$ & IgE & Immunglobulin E (HP) & $\mathrm{IU} / \mathrm{ml}$ & $\mathrm{kU} / 1$ \\
\hline
\end{tabular}

\section{Discussion and Conclusion}

Differences in site-specific reporting are expected but can't be eliminated solely by mapping to LOINC. Therefore, using Unified Code for Units of Measure (UCUM) can significantly improve semantic interoperability. Employing UCUM would not only eliminate minor disparities as described in category 1) but could also enable the automated conversion between related units differing in granularity [5].

\section{Acknowledgment}

The project is funded by the German Federal Ministry of Education and Research (BMBF, grant id: 01ZZ1802U).

\section{References}

[1] Semler SC, Wissing F, and Heyder R. German Medical Informatics Initiative. Methods Inf. Med., 2018; 57(1); e50-e56, 2018.

[2] Semler SC. LOINC: Origin, development of and perspectives for medical research and biobanking - 20 years on the way to implementation in Germany. J. Lab. Med. 2019; 43(6): 359-382, 2019, doi: 10.1515/labmed-2019-0193.

[3] Drenkhahn C and Ingenerf J. The LOINC Content Model and Its Limitations of Usage in the Laboratory Domain, Stud. Health Technol. Inform. 2020; 270: 437-442, Jun. 2020, doi: 10.3233/SHTI200198.

[4] "RELMA," LOINC. https://loinc.org/relma/ (accessed Jul. 15, 2020).

[5] Hauser RG, Quine DB, Ryder A, Campbell S. Unit conversions between LOINC codes. J. Am. Med. Inform. Assoc. JAMIA. 2017; 25(2): 192-196, Jun. 2017, doi: 10.1093/jamia/ocx056. 